\title{
THE FIELD OF A STEP-LIKE ACCELERATED POINT CHARGE: LIÉNARD-WIECHERT POTENTIALS AND ČERENKOV SHOCK WAVES REVISITED
}

\author{
L'ubomír Šumichrast ${ }^{*}$
}

\begin{abstract}
Scalar and vector potential as well as the electromagnetic field of a moving point charge is a nice example how the application of symbolic functions (distributions) in electromagnetics makes it easier to obtain and interpret solutions of otherwise hardly solvable problems.
\end{abstract}

K e y w or d s: scalar potential, vector potential

\section{INTRODUCTION}

Electromagnetic field of the moving point charge is in the literature commonly expressed in form of the LiénardWiechert potentials $[1-3]$. In the case of the charge moving along the straight line with the constant velocity the pertaining expressions can be obtained also by direct application of the relativistic Lorentz transformation of the Coulomb potential of moving charge [4]. It is shown here that the respective results can by obtained by straightforward application of the formalism of symbolic functions (distributions) using the classical formulae for retarded potentials. It is shown that the application of this formalism makes it possible, by relatively simple means, to solve also the case of the electromagnetic field of the steplike accelerated charge.

It is well-known that the charge moving with velocity $v$ in the medium where the velocity of light is $c=1 / \sqrt{\mu \varepsilon}$, provided $v<c$, does not radiate electromagnetic energy in spite of the fact that the electromagnetic field changes in time due to the moving charge.

In the case $v>c$, that is of course impossible in vacuum, where $c=c_{0}=1 / \sqrt{\mu_{0} \varepsilon_{0}}$, but can well occur in material media - in the theory modeled by a continuum with $c=1 / \sqrt{\mu \varepsilon}<c_{0}$ - the electromagnetic energy is out-radiated in form of a Čerenkov shock wave similar to sonic shock wave arising when a plane overcomes the sonic barrier - the sound velocity in air. Here this difference in non-propagating waves connected with the charge moving at the velocity $v<c$ and Cerenkov radiation for $v>c$ is shown using the expansion of the field into the spectrum of plane, or cylindrical waves - in the first case nonpropagating evanescent waves bound to the charge and in the second the waves radiating energy away from the charge.

\section{RETARDED POTENTIALS}

In many textbooks on electromagnetics eg [1-3] the formulae for the retarded scalar potential $\Phi(\boldsymbol{r}, t)$, and retarded vector potential $\boldsymbol{A}(\boldsymbol{r}, t)$ in the infinite homogeneous space with constant permittivity $\varepsilon$ and constant permeability $\mu$, are often commonly simply written down in the form

$$
\begin{aligned}
& \Phi(\boldsymbol{r}, t)=\frac{1}{4 \pi \varepsilon} \iiint_{\infty} \frac{q\left(\mathbf{r}^{\prime}, t-\left|\boldsymbol{r}-\mathbf{r}^{\prime}\right| / c\right)}{\left|\mathbf{r}-\mathbf{r}^{\prime}\right|} \mathrm{d} v^{\prime}, \\
& \boldsymbol{A}(\boldsymbol{r}, t)=\frac{\mu}{4 \pi} \iiint_{\infty} \frac{\boldsymbol{J}\left(\mathbf{r}^{\prime}, t-\left|\boldsymbol{r}-\mathbf{r}^{\prime}\right| / c\right.}{\left|\boldsymbol{r}-\mathbf{r}^{\prime}\right|} \mathrm{d} v^{\prime},
\end{aligned}
$$

where $\boldsymbol{r}$ is the observation point, $\boldsymbol{r}^{\prime}$ the source point, $q\left(\mathbf{r}^{\prime}, t\right)$ is the charge density and $\boldsymbol{J}\left(\boldsymbol{r}^{\prime}, t\right)$ the current density. The retardation in time is given by the term $\left|\boldsymbol{r}-\mathbf{r}^{\prime}\right| / c$, where $c=1 / \sqrt{\mu \varepsilon}$ is the light velocity in a given medium, ie the time needed for any information to propagate along the path $\left|\boldsymbol{r}-\boldsymbol{r}^{\prime}\right|$ with the velocity $c$.

Formulae (1) and (2) are solutions of the wave equations

$$
\begin{gathered}
\nabla^{2} \Phi(\boldsymbol{r}, t)-\frac{1}{c^{2}} \frac{\partial^{2} \Phi(\boldsymbol{r}, t)}{\partial t^{2}}=-q(\boldsymbol{r}, t) / \varepsilon, \\
\nabla^{2} \boldsymbol{A}(\boldsymbol{r}, t)-\frac{1}{c^{2}} \frac{\partial^{2} \boldsymbol{A}(\boldsymbol{r}, t)}{\partial t^{2}}=-\mu \boldsymbol{J}(\boldsymbol{r}, t),
\end{gathered}
$$

obtained from Maxwell's equations using the Lorentz gauge. Here $\nabla^{2}$ is the Laplace operator. The field quantities are then obtained in the usual way as

$$
\begin{gathered}
\boldsymbol{E}(\boldsymbol{r}, t)=-\operatorname{grad} \Phi(\boldsymbol{r}, t)-\partial \boldsymbol{A}(\boldsymbol{r}, t) / \partial t, \\
\boldsymbol{B}(\boldsymbol{r}, t)=\operatorname{curl} \boldsymbol{A}(\boldsymbol{r}, t)
\end{gathered}
$$

The above formulation (1) and (2) means in fact the space convolution after the the time convolution of the

* Slovak University of Technology, Institute of Electrical Engineering Ilkovičova 3, SK-81219 Bratislava, Slovakia lubomir.sumichrast@stuba.sk 
pertaining source densities $q(\boldsymbol{r}, t)$ and $\boldsymbol{J}(\boldsymbol{r}, t)$ with the Green function of the infinite homogeneous space $G(\mathbf{r}, t)$ has been performed, ie

$\Phi(\boldsymbol{r}, t)=\frac{1}{4 \pi \varepsilon} \iiint_{\infty}\left[\int_{-\infty}^{\infty} q\left(\mathbf{r}^{\prime}, \tau\right) G\left(\boldsymbol{r}-\mathbf{r}^{\prime}, t-\tau\right) d \tau\right] \mathrm{d} v^{\prime}$

$\boldsymbol{A}(\boldsymbol{r}, t)=\frac{\mu}{4 \pi} \iiint_{\infty}\left[\int_{-\infty}^{\infty} \boldsymbol{J}\left(\mathbf{r}^{\prime}, \tau\right) G\left(\boldsymbol{r}-\mathbf{r}^{\prime}, t-\tau\right) \mathrm{d} \tau\right] \mathrm{d} v^{\prime}$

The Green function $G(\boldsymbol{r}, t)$ of the infinite homogeneous space is given as the solution of the wave equation

$$
\nabla^{2} G(\boldsymbol{r}, t)-\frac{1}{c^{2}} \frac{\partial^{2} G(\boldsymbol{r}, t)}{\partial t^{2}}=-\delta(\boldsymbol{r}) \delta(t)
$$

on an infinite domain, where $\delta(t)$ means the Dirac deltafunction and $\delta(\mathbf{r})=\delta(x) \delta(y) \delta(z)$ the three dimensional delta-function. The solution of (9) can be easily obtained eg [5] in form of the spherical delta-function wave-front spreading in space with velocity $c$ as a function of time,

$$
G(\boldsymbol{r}, t)=G(r, t)=\delta(t-r / c) / 4 \pi r,
$$

where $r=|\boldsymbol{r}|=\sqrt{x^{2}+y^{2}+z^{2}}$. Thus substituting (10) into (7) and (8) leads to (1) and (2).

\section{RETARDED POTENTIALS FOR THE MOVING POINT CHARGE}

The charge and current density of a point charge moving with the velocity $v$ (for simplicity) in direction of $z$ axis can be easily expressed using the delta-functions

$$
\begin{gathered}
q(\boldsymbol{r}, t)=Q \delta(x) \delta(y) \delta(z-v t), \\
\boldsymbol{J}(\boldsymbol{r}, t)=Q v \delta(x) \delta(y) \delta(z-v t) \mathbf{u}_{z},
\end{gathered}
$$

or simply $J_{z}(\boldsymbol{r}, t)=v q(\boldsymbol{r}, t)$. After having performed at first the space convolution in (7) and (8) one obtains

$$
\begin{aligned}
\Phi(\boldsymbol{r}, t)= & \frac{c^{2}}{v} A_{z}(\boldsymbol{r}, t)= \\
& \frac{Q}{4 \pi \varepsilon} \int_{-\infty}^{\infty} \frac{\delta\left(t-\tau-\sqrt{\rho^{2}+(z-v \tau)^{2}} / c\right)}{\sqrt{\rho^{2}+(z-v \tau)^{2}}} \mathrm{~d} \tau,
\end{aligned}
$$

where the common cylindrical coordinate system has been introduced, with the variables and the unit vectors

$$
\begin{gathered}
\rho=\sqrt{x^{2}+y^{2}}, \quad \psi=\arctan (y / x), \\
\mathbf{u}_{\rho}=\mathbf{u}_{x} \cos \psi+\mathbf{u}_{y} \sin \psi, \quad \mathbf{u}_{\psi}=-\mathbf{u}_{x} \sin \psi+\mathbf{u}_{y} \cos \psi,
\end{gathered}
$$

and with the reverse relation $x=\rho \cos \psi, y=\rho \sin \psi$.

To solve (13) one may reformulate the integral therein

$$
\int_{-\infty}^{\infty} \frac{\delta(t-\ldots)}{\sqrt{\cdots}} \mathrm{d} \xi=\frac{\mathrm{d}}{\mathrm{d} t} \int_{-\infty}^{\infty} \frac{\mathbf{1}(t-\ldots)}{\sqrt{\cdots}} \mathrm{d} \tau,
$$

where $\mathbf{1}(t-\ldots)$ is the unit-step-function (Heaviside function). After the substitution $t-\tau=\xi$ this integral takes the form

$$
\frac{\mathrm{d}}{\mathrm{d} t} \int_{-\infty}^{\infty} \frac{\mathbf{1}\left(\xi-\sqrt{\rho^{2}+(z-v t+v \xi)^{2}} / c\right)}{\sqrt{\rho^{2}+(z-v t+v \xi)^{2}}} \mathrm{~d} \xi
$$

Since the square root in (15) is always positive (physically it has a meaning of distance) the value of the variable $\xi$ in the argument of the delta-function must be positive too. This leads to the positive root

$$
\xi_{0}=\frac{v(z-v t)+\sqrt{c^{2}(z-v t)^{2}+\left(c^{2}-v^{2}\right) \rho^{2}}}{c^{2}-v^{2}}
$$

of the quadratic equation

$$
\left(c^{2}-v^{2}\right) \xi^{2}-2 v(z-v t) \xi-\rho^{2}-(z-v t)^{2}=0,
$$

pertaining to the zero point of the argument of the unitstep-function in (15).

Then integral

$$
\frac{\mathrm{d}}{\mathrm{d} t} \int_{\xi_{0}}^{\infty} \frac{1}{\sqrt{\rho^{2}+(z-v t+v \xi)^{2}}} \mathrm{~d} \xi
$$

yields with use of the formula 2.261 in [6] the final result

$$
\left[\left(1-v^{2} / c^{2}\right) \rho^{2}+(z-v t)^{2}\right]^{-1 / 2}
$$

4 CASE $v<c$ :

\section{LIÉNARD-WIECHERT POTENTIALS}

The potentials for the moving point charge — called Liénard-Wiechert potentials — are thus given as

$$
\Phi(\mathbf{r}, t)=\frac{c^{2}}{v} A_{z}(\mathbf{r}, t)=\frac{Q}{4 \pi \varepsilon} \frac{1}{\sqrt{\eta \rho^{2}+(z-v t)^{2}}},
$$

where $\eta=1-(v / c)^{2}$ is the Lorentz factor and (20) is the relastivistic Lorentz transformation of the electrostatic scalar potential. The resulting electric field intensity of a moving point charge equals

$$
\boldsymbol{E}(\boldsymbol{r}, t)=\frac{Q}{4 \pi \varepsilon} \eta \frac{\rho \boldsymbol{u}_{\rho}+(z-v t) \mathbf{u}_{z}}{\left[\eta \rho^{2}+(z-v t)^{2}\right]^{3 / 2}},
$$

with the conservative part

$$
-\operatorname{grad} \Phi(\mathbf{r}, t)=\frac{Q}{4 \pi \varepsilon} \frac{\eta \rho \mathbf{u}_{\rho}+(z-v t) \mathbf{u}_{z}}{\left[\eta \rho^{2}+(z-v t)^{2}\right]^{3 / 2}},
$$




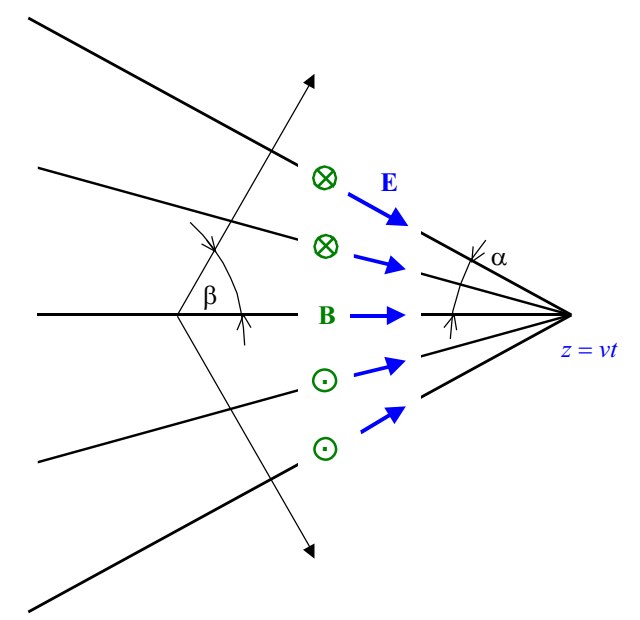

Fig. 1. Conical domain and field orientatoion for the Cerenkov radiation

and the solenoidal part

$$
-\frac{\partial \boldsymbol{A}(\boldsymbol{r}, t)}{\partial t}=-\frac{Q}{4 \pi \varepsilon} \frac{(v / c)^{2}(z-v t) \mathbf{u}_{z}}{\left[\eta \rho^{2}+(z-v t)^{2}\right]^{3 / 2}} .
$$

The magnetic field induction of a moving point charge reads

$$
\boldsymbol{B}(\mathbf{r}, t)=\frac{\mu}{4 \pi} Q v \frac{\eta \rho \boldsymbol{u}_{\psi}}{\left[\eta \rho^{2}+(z-v t)^{2}\right]^{3 / 2}} .
$$

Notice that the star-shaped field lines of the electric field $\boldsymbol{E}(\boldsymbol{r}, t)$ accordingly (21) are in any time instant the same as for the electrostatic field, but the field itself is moving, it has the conservative as well as the solenoidal component. The equipotential surfaces of $\Phi(\boldsymbol{r}, t)$ are not spherical but ellipsoidal surfaces moving together with the charge.

The Poynting vector $\boldsymbol{P}=\boldsymbol{E} \times \boldsymbol{B} / \mu$ representing the power flow density in the electromagnetic field reads

$$
\boldsymbol{P}(\boldsymbol{r}, t)=\frac{Q^{2} v}{16 \pi^{2} \varepsilon} \frac{\eta^{2} \rho\left[\rho \mathbf{u}_{z}-(z-v t) \mathbf{u}_{\rho}\right]}{\left[\eta \rho^{2}+(z-v t)^{2}\right]^{3}} .
$$

In fact the power flow density is circulating around the charge and this circulation is moving with it since the expresion $\left[\rho \boldsymbol{u}_{z}-(z-v t) \boldsymbol{u}_{\rho}\right]=-\boldsymbol{u}_{\vartheta} \sqrt{\rho^{2}+(z-v t)^{2}}$, where $\boldsymbol{u}_{\vartheta}$ is the unit vector of the local spherical coordinate system (moving with the charge) pertaining to the polar angle $\vartheta$. The steady power density flow occurs in the direction of the $z$-axis only and in the plane of the moving charge it reads

$$
\left.P_{z}\right|_{z=v t}=\frac{Q^{2} v}{16 \pi^{2} \varepsilon\left(1-v^{2} / c^{2}\right) \rho^{4}} .
$$

\section{CASE $v>c$ : ČERENKOV SHOCK WAVE}

Velocity of the moving charge can never be higher than the velocity of light in vacuum $c_{0}=1 / \sqrt{\mu_{0} \varepsilon_{0}}$. However, in the material media represented by continuum with $\mu>$ $\mu_{0}$ and $\varepsilon>\varepsilon_{0}$ is the phase velocity of the propagating plane wave $c=1 / \sqrt{\mu \varepsilon}$ smaller than $c_{0}$ and in principle the velocity of the moving charge can be higher than $c$.

In such a case the value under the square root in (16) is positive only for the relation

$$
v t-z>\rho \sqrt{v^{2} / c^{2}-1}
$$

The domain defined by (27) is the conical domain to the left from the conus vertex point $z=v t$ and with the conus-opening-angle $\alpha$, where $\sin \alpha=c / v$ as shown in Fig. 1. The conical boundary spreads out in direction given by the angle $\beta, \cos \beta=c / v$. The solution outside this domain is zero, therefore (20) must be written in the form

$$
\Phi(\boldsymbol{r}, t)=\frac{c^{2}}{v} A_{z}(\boldsymbol{r}, t)=\frac{Q}{4 \pi \varepsilon} \frac{\mathbf{1}(v t-z-\rho \sqrt{\chi})}{\sqrt{(v t-z)^{2}-\chi \rho^{2}}},
$$

where $\chi=-\eta=(v / c)^{2}-1$, yielding thus the electric field intensity

$$
\begin{aligned}
\boldsymbol{E}(\boldsymbol{r}, t)= & \frac{Q}{4 \pi \varepsilon} \chi \frac{-\rho \boldsymbol{u}_{\rho}+(v t-z) \mathbf{u}_{z}}{\left[(v t-z)^{2}-\chi \rho^{2}\right]^{3 / 2}} \mathbf{1}(v t-z-\rho \sqrt{\chi}) \\
& +\frac{Q}{4 \pi \varepsilon} \frac{\boldsymbol{u}_{\rho} \sqrt{\chi}-\chi \mathbf{u}_{z}}{\sqrt{(v t-z)^{2}-\chi \rho^{2}}} \delta(v t-z-\rho \sqrt{\chi})
\end{aligned}
$$

and the magnetic field induction

$$
\begin{array}{rl}
\boldsymbol{B}(\boldsymbol{r}, t)=-\frac{\mu}{4 \pi} Q & v \frac{\mathbf{1}(v t-z-\rho \chi)}{\left[(v t-z)^{2}-\rho \chi^{2}\right]^{3 / 2}} \chi \rho \mathbf{u}_{\psi} \\
+ & \frac{\mu}{4 \pi} Q v \frac{\delta(v t-z-\rho \sqrt{\chi})}{\sqrt{(v t-z)^{2}-\chi \rho^{2}}} \sqrt{\chi} \mathbf{u}_{\psi} .
\end{array}
$$

It can be easily seen that the directions of the regular terms of the electric and magnetic field are reversed as compared to (21) and (24). The $\delta$-like terms on the conical boundary represent the shock wave.

The regular part of the pointing vector within the conical domain reads

$$
\boldsymbol{P}(\boldsymbol{r}, t)=\frac{Q^{2} v}{16 \pi^{2} \varepsilon} \frac{\chi^{2} \rho\left[\rho \mathbf{u}_{z}+(v t-z) \mathbf{u}_{\rho}\right]}{\left[(v t-z)^{2}-\chi \rho^{2}\right]^{3}} .
$$

On the conical boundary enclosing the electromagnetic field the Poynting vector $\boldsymbol{P}(\boldsymbol{r}, t)$ is directed outwards and perpendicular to this boundary.

Observe that if the charge moves with exactly the transitional velocity $v=c$, ie $\chi=-\eta=0$ then in (28)

$$
\Phi(\boldsymbol{r}, t)=\frac{c^{2}}{v} A_{z}(\mathbf{r}, t)=\frac{Q}{4 \pi \varepsilon} \frac{\mathbf{1}(v t-z)}{v t-z}
$$

and this theory leads to $\boldsymbol{E}(\boldsymbol{r}, t)=0, \boldsymbol{B}(\boldsymbol{r}, t)=0$. 


\section{SPECTRAL EXPANSION}

When spatio-temporal spectra of the charge and curent density is introduced one obtains after the temporal and two-dimensional spatial Fourier transform of (11) for $q\left(x, \kappa_{y}, \kappa_{z}, \omega\right)$

$$
q\left(x, \kappa_{y}, \kappa_{z}, \omega\right)=2 \pi Q \delta(x) \delta\left(\omega-\kappa_{z} v\right),
$$

and similarly for $J_{z}\left(x, \kappa_{y}, \kappa_{z}, \omega\right)=v q\left(x, \kappa_{y}, \kappa_{z}, \omega\right)$. After the substitution into (65) and integration with respect to $x^{\prime}$ and $\kappa_{z}$ one obtains for temporal frequency spectra $\Phi(\boldsymbol{r}, \omega)$ and $A_{z}(\boldsymbol{r}, \omega)$ the formula

$$
\begin{aligned}
& \Phi(\boldsymbol{r}, \omega)=\frac{c^{2}}{v} A_{z}(\boldsymbol{r}, \omega)=\frac{j Q}{4 \pi \varepsilon v} \times \\
& \int_{-\infty}^{\infty} \frac{\exp \left(j|x| \sqrt{k_{0}^{2}-k^{2}-\kappa_{y}^{2}}\right)}{\sqrt{k_{0}^{2}-k^{2}-\kappa_{y}^{2}}} \exp \left(j \kappa_{y} y\right) \exp (j k z) \mathrm{d} \kappa_{y},
\end{aligned}
$$

where $k_{0}=\omega / c$ and $k=\omega / v$.

For the charge moving with the velocity $v$, for the classical case $v<c$, ie $k>k_{0},(34)$ takes the form

$$
\begin{aligned}
& \Phi(\boldsymbol{r}, \omega)=\frac{c^{2}}{v} A_{z}(\boldsymbol{r}, \omega)=\frac{Q}{4 \pi \varepsilon v} \times \\
& \int_{-\infty}^{\infty} \frac{\exp \left(-|x| \sqrt{k^{2}-k_{0}^{2}+\kappa_{y}^{2}}\right)}{\sqrt{k^{2}-k_{0}^{2}+\kappa_{y}^{2}}} \exp \left(j \kappa_{y} y\right) \exp (j k z) \mathrm{d} \kappa_{y} .
\end{aligned}
$$

In this case the spectrum consists of the evanescent waves only.

The integration in (35) can be performed [7] with the result

$$
\begin{aligned}
\Phi(\boldsymbol{r}, \omega)=\frac{c^{2}}{v} A_{z}(\boldsymbol{r}, \omega) \\
\quad=\frac{Q}{2 \pi \varepsilon v} K_{0}\left(\rho \sqrt{k^{2}-k_{0}^{2}}\right) \exp (j k z),
\end{aligned}
$$

where $K_{0}$ is the modified Bessel function of second kind, representing cylindrical evanescent waves propagating, $i e$ transmitting the energy, in the $z$-direction only, with exponentially attenuated amplitude for $\rho \rightarrow \infty$. The inverse Fourier transform in time domain yields the formula (20).

For the Čerenkov shock wave $v>c$ holds, ie $k<$ $k_{0}$. The expression in the integral (34) represents until $\kappa_{y}^{2}<k_{0}^{2}-k^{2}$ the propagating homogeneous plane waves in directions

$$
\pm \boldsymbol{u}_{x} \sqrt{k_{0}^{2}-k^{2}-\kappa_{y}^{2}}+\mathbf{u}_{y} \kappa_{y}+\mathbf{u}_{z} k
$$

given by the unit vectors $\left[\boldsymbol{u}_{x}, \boldsymbol{u}_{y}, \boldsymbol{u}_{z}\right]$, with the phase velocity $c=\omega / k_{0}$. All of the them span exactly the angle $\beta$ with the $z$-axis, where $\tan \beta=\sqrt{k_{0}^{2}-k^{2}} / k$, or $\cos \beta=c / v$, and this is exactly the direction in that the conical boundary of the shock wave spreads out in space with velocity $c$ as shown in previous paragraph and in
Fig. 1. This is the direction of the power flow density, $i e$ the direction in which the energy of the Cerenkov radiation is out-radiated. For $\kappa_{y}^{2}>k_{0}^{2}-k^{2}$ one arrives again to the spectral components in form of evanescent waves.

Formula [34] can be integrated too [7], with the result

$$
\begin{aligned}
\Phi(\boldsymbol{r}, \omega)=\frac{c^{2}}{v} A_{z}(\boldsymbol{r}, \omega) \\
\quad=\frac{j Q}{4 \pi \varepsilon v} H_{0}^{(1)}\left(\rho \sqrt{k_{0}^{2}-k^{2}}\right) \exp (j k z),
\end{aligned}
$$

where $H_{0}^{(1)}$ is the Hankel function with an oscillatory character. Here the energy is not only transmitted along the $z$-axis but also out-radiated in the cylindrical radial direction.

\section{POTENTIALS OF THE STEP-LIKE ACCELERATED POINT CHARGE}

Let us consider the modified situation when the point charge is up to $t=0$ at rest and for $t>0$ it will be stepwise accelerated to the velocity $v$, ie in the direction of the $z$-axis. Hence

$$
\begin{gathered}
q(\boldsymbol{r}, t)=Q \delta(x) \delta(y) \delta(z-v t \mathbf{1}(t)), \\
\boldsymbol{J}(\boldsymbol{r}, t)=Q v \delta(x) \delta(y) \delta(z-v t \mathbf{1}(t)) \mathbf{1}(t) \mathbf{u}_{z}
\end{gathered}
$$

The current density $\boldsymbol{J}(\boldsymbol{r}, t)$ is obviously equal to zero for $t<0$ when the charge is at rest. The formulae for the retarded potentials now read

$$
\begin{aligned}
\Phi(\boldsymbol{r}, t)= & \frac{Q}{4 \pi \varepsilon} \times \\
& \int_{-\infty}^{\infty} \frac{\delta\left(t-\tau-\sqrt{\rho^{2}+[z-v \tau \mathbf{1}(\tau)]^{2}} / c\right)}{\sqrt{\rho^{2}+[z-v \tau \mathbf{1}(\tau)]^{2}}} \mathrm{~d} \tau,
\end{aligned}
$$

$$
\begin{aligned}
& A_{z}(\boldsymbol{r}, t)=\frac{\mu}{4 \pi} Q v \times \\
& \int_{-\infty}^{\infty} \frac{\delta\left(t-\tau-\sqrt{\rho^{2}+[z-v \tau \mathbf{1}(\tau)]^{2}} / c\right)}{\sqrt{\rho^{2}+[z-v \tau \mathbf{1}(\tau)]^{2}}} \mathbf{1}(\tau) \mathrm{d} \tau,
\end{aligned}
$$

As long as $t<0$ holds, the arguments of $\delta$-functions can be equal to zero only for $\tau<0$, yielding $\tau \mathbf{1}(\tau)=0$. Therefore for $t<0$ the vector potential is zero $\boldsymbol{A}(\boldsymbol{r}, t)=$ 0 - there is no current, the charge is at rest, and the scalar potential

$$
\Phi(\boldsymbol{r}, t)=\frac{Q}{4 \pi \varepsilon} \int_{-\infty}^{\infty} \frac{\delta\left(t-\tau-\sqrt{\rho^{2}+z^{2}} / c\right)}{\sqrt{\rho^{2}+z^{2}}} \mathrm{~d} \tau=\frac{Q}{4 \pi \varepsilon r}
$$

is identical with the electrostatic potential of the point charge. 


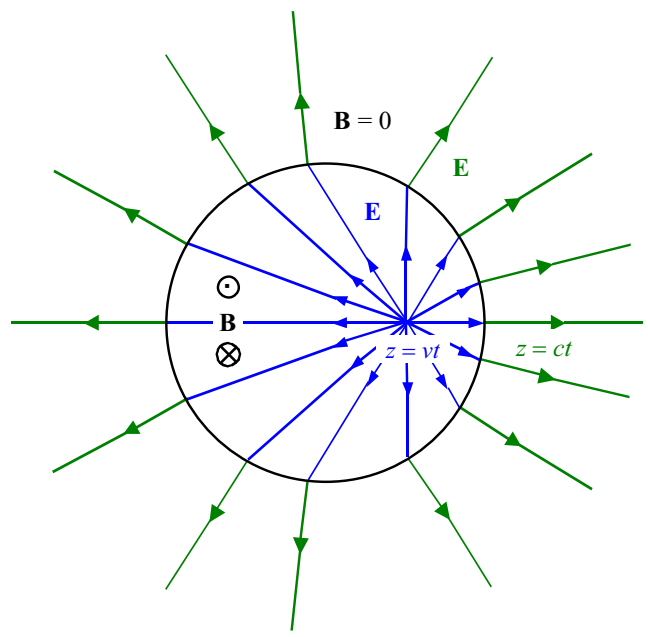

Fig. 2. Transient domain between the field of moving charge with $v<c$ and the electrostatic field

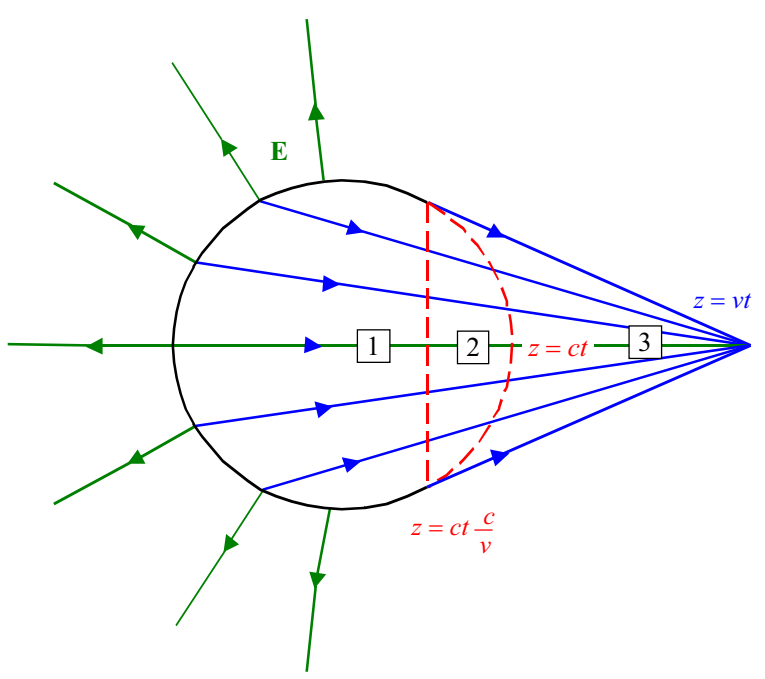

Fig. 3. Transient domain between the field of moving charge with $v>c$ (Čerenkov shock wave) and the electrostatic field

For $t>0$ and $\tau>0$ the formulae for the retarded potentials are the same as (13). For the variable $\xi$ in the additional condition $\tau=t-\xi>0$, ie $\xi<t$, due to the presence of the unit-step-function $\mathbf{1}(\tau)$ in (41) and (42) must be met. Hence for $v<c$, the variable $\xi_{0}$ in (16) must fulfil the condition $\xi_{0}<t$ leading to the relation

$$
\sqrt{c^{2}(z-v t)^{2}+\left(c^{2}-v^{2}\right) \rho^{2}}<c^{2} t-v z,
$$

which can be easily recast into the simple form

$$
\sqrt{z^{2}+\rho^{2}}<c t
$$

The right hand side of (44) must itself fulfil the condition $z<c t(c / v)$. This together leads to the spatiotemporal domain of validity in the form

$$
z<c t(c / v) \cap \sqrt{z^{2}+\rho^{2}}<c t .
$$

Since in this case $c / v>1$ the solution is $\sqrt{z^{2}+\rho^{2}}<c t$, $i e$ it represents the inner domain of the sphere with the in-time-expanding radius $r=c t$ as shown in Fig. 2 .
The field inside the sphere, $\sqrt{z^{2}+\rho^{2}}<c t$, is identical with the electric and the magnetic field of the moving charge (21) and (24). The field outside $\sqrt{z^{2}+\rho^{2}}>c t$ is the electrostatic field

$$
\boldsymbol{E}(\boldsymbol{r}, t)=\frac{Q}{4 \pi \varepsilon} \frac{\rho \boldsymbol{u}_{\rho}+z \boldsymbol{u}_{z}}{\left[\rho^{2}+z^{2}\right]^{3 / 2}}=\frac{Q}{4 \pi \varepsilon r^{2}} \mathbf{u}_{r} .
$$

Delta-like terms of the fields exist on the spherical boundary surface $r=c t$ in this case too and are given by

$$
\begin{gathered}
\left.\boldsymbol{E}(r, \vartheta, t)\right|_{r=c t}=\frac{Q}{4 \pi \varepsilon c t} \frac{(v / c) \cos \vartheta}{1-(v / c) \cos \vartheta}\left(\mathbf{u}_{r}+\mathbf{u}_{z}\right) \delta(r-c t) \\
\left.\boldsymbol{B}(r, \vartheta, t)\right|_{r=c t}=-\frac{\mu Q v}{4 \pi c t} \frac{1}{1-(v / c) \cos \vartheta} \mathbf{u}_{\psi} \delta(r-c t)
\end{gathered}
$$

For the case $v>c$ the solution can exist only within the volume of the conical domain (50). If the conditions $\xi>\xi_{0}, \xi<t$ should hold, the inequality

$$
\sqrt{c^{2}(v t-x)^{2}-\rho^{2}\left(v^{2}-c^{2}\right)}<v z-c^{2} t
$$

has to be met. If the right hand side of (50) is positive, $i e$ if $z>c t(c / v)$ then (50) is fulfilled in the domain

$$
(v t-z)>\rho \sqrt{\left(v^{2} / c^{2}\right)-1} \cap \sqrt{\rho^{2}+z^{2}}>c t .
$$

This is the spatio-temporal domain of the conus to the right outside of the sphere $\sqrt{z^{2}+\rho^{2}}<c t$ as seen in Fig. 3. Together with (46) this leads to the domain of existence of the wave depicted in Fig. 3.

\section{CONCLUSIONS}

The analysis presented here demonstrates that by using the formalism of Heaviside unit-step function and the $\delta$-function as a derivative of the Heaviside function makes it possible to obtain the results representing the relativistic character of electric and magnetic field by purely classical approach. For the step-like accelerated point charge the shape of the boundary between the electrostatic field of the charge at rest and the dynamic field of the moving charge has been uniquely identified. This paper is a revised version of the author's work [8].

\section{Appendix}

Let the Fourier transform in time domain be

$$
f(\omega)=\int_{-\infty}^{\infty} f(t) \exp (j \omega t) \mathrm{d} t
$$

then the temporal spectrum $G(\boldsymbol{r}, \omega)$ of the Green function $G(\boldsymbol{r}, t)=\delta(t-r / c) / 4 \pi r$ in (10) is given by the Helmholtz equation with $k_{0}=\omega / c$, and solution

$$
\nabla^{2} G(\mathbf{r}, \omega)+k_{0}^{2} G(\mathbf{r}, \omega)=-\delta(\mathbf{r}),
$$




$$
G(\boldsymbol{r}, \omega)=\exp \left(j k_{0} r\right) / 4 \pi r .
$$

The spectral densities $\Phi(\boldsymbol{r}, \omega), \boldsymbol{A}(\boldsymbol{r}, \omega)$ of the scalar and vector potential $\Phi(\boldsymbol{r}, t), \boldsymbol{A}(\boldsymbol{r}, t)$ are then expressed in the form

$$
\begin{aligned}
& \Phi(\boldsymbol{r}, \omega)=\frac{1}{4 \pi \varepsilon} \iiint_{\infty} q\left(\boldsymbol{r}^{\prime}, \omega\right) \frac{\exp \left(j k_{0}\left|\boldsymbol{r}-\boldsymbol{r}^{\prime}\right|\right)}{\left|\boldsymbol{r}-\mathbf{r}^{\prime}\right|} \mathrm{d} v^{\prime} \\
& \boldsymbol{A}(\boldsymbol{r}, \omega)=\frac{\mu}{4 \pi} \iiint_{\infty} \boldsymbol{J}\left(\boldsymbol{r}^{\prime}, \omega\right) \frac{\exp \left(j k_{0}\left|\boldsymbol{r}-\boldsymbol{r}^{\prime}\right|\right)}{\left|\boldsymbol{r}-\boldsymbol{r}^{\prime}\right|} \mathrm{d} v^{\prime}
\end{aligned}
$$

If subsequently the two-dimensional Fourier transform with respect to spatial variables $y, z$ is introduced, ie

$$
\begin{aligned}
& f\left(x, \kappa_{y}, \kappa_{z}, \omega\right)= \\
& \qquad \iint_{\infty} f(x, y, z, \omega) \exp \left[-j\left(\kappa_{y} y+\kappa_{z} z\right)\right] \mathrm{d} y \mathrm{~d} z,
\end{aligned}
$$

then transformed Helmholtz equation (53) reads

$$
\frac{\mathrm{d}^{2} G\left(x, \kappa_{y}, \kappa_{z}, \omega\right)}{\mathrm{d} x^{2}}+\left(k_{0}^{2}-\kappa_{y}^{2}-\kappa_{y}^{2}\right) G\left(x, \kappa_{y}, \kappa_{z}, \omega\right)=-\delta(x) .
$$

General solution of the homogeneous equation (58), ie of equation (58) with zero right hand side, equals

$$
\begin{array}{r}
G\left(x, \kappa_{y}, \kappa_{z}, \omega\right)=A \exp \left(j x \sqrt{k_{0}^{2}-\kappa_{y}^{2}-\kappa_{y}^{2}}\right)+ \\
B \exp \left(-j x \sqrt{k_{0}^{2}-\kappa_{y}^{2}-\kappa_{y}^{2}}\right) .
\end{array}
$$

Taking into account the radiation condition the first term is valid for $x>0$ only and the second for $x<0$. This can be generally written as

$$
\begin{gathered}
G\left(x, \kappa_{y}, \kappa_{z}, \omega\right)=A \exp \left(j x \sqrt{k_{0}^{2}-\kappa_{y}^{2}-\kappa_{y}^{2}}\right) \mathbf{1}(x)+ \\
B \exp \left(-j x \sqrt{k_{0}^{2}-\kappa_{y}^{2}-\kappa_{y}^{2}}\right) \mathbf{1}(-x) . \\
\mathrm{d} G / \mathrm{d} x=(A-B) \delta(x)+ \\
j \sqrt{\cdots}\{A \exp (j x \sqrt{\cdots}) \mathbf{1}(x)-B \exp (-j x \sqrt{\cdots}) \mathbf{1}(-x)\},
\end{gathered}
$$

yielding $A=B$, since the delta term must cancel out.

$$
\begin{aligned}
& \mathrm{d}^{2} G / \mathrm{d} x^{2}=+2 j A \sqrt{k_{0}^{2}-\kappa_{y}^{2}-\kappa_{y}^{2}} \delta(x)-A\left[k_{0}^{2}-\kappa_{y}^{2}-\kappa_{z}^{2}\right] \\
& \quad \times\{\exp (j x \sqrt{\cdots}) \mathbf{1}(x)-\exp (-j x \sqrt{\cdots}) \mathbf{1}(-x)\} .
\end{aligned}
$$

This after substitution into (58) leads to the result

$$
A=j / 2 \sqrt{k_{0}^{2}-\kappa_{y}^{2}-\kappa_{y}^{2}},
$$

$$
G\left(x, \kappa_{y}, \kappa_{z}, \omega\right)=\frac{j \exp \left(j|x| \sqrt{k_{0}^{2}-\kappa_{y}^{2}-\kappa_{y}^{2}}\right)}{2 \sqrt{k_{0}^{2}-\kappa_{y}^{2}-\kappa_{y}^{2}}} .
$$

For the two-dimensional spatio-temporal spectral density of the scalar potential one thus obtains

$$
\begin{gathered}
\Phi(\boldsymbol{r}, \omega)=\frac{j}{8 \pi^{2} \varepsilon} \int_{-\infty}^{\infty}\left\{\iint_{\infty} q\left(x^{\prime}, \kappa_{y}, \kappa_{z}, \omega\right)\right. \\
\times \frac{\exp \left(j\left|x-x^{\prime}\right| \sqrt{k_{0}^{2}-\kappa_{y}^{2}-\kappa_{y}^{2}}\right)}{\sqrt{k_{0}^{2}-\kappa_{y}^{2}-\kappa_{y}^{2}}} \\
\left.\exp \left[j\left(\kappa_{y} y+\kappa_{z} z\right)\right] \mathrm{d} \kappa_{y} \mathrm{~d} \kappa_{z}\right\} \mathrm{d} x^{\prime} .
\end{gathered}
$$

Similar formula can be found for the vector potential $\boldsymbol{A}(\boldsymbol{r}, \omega)$ of current density too.

\section{Acknowledgement}

This work was supported by the Slovak Research and Development Agency under the contract No. APVV0062-11.

\section{REFERENCES}

[1] TRALLI, N.: Classical Electromagnetic Theory, McGraw Hill, New York, 1963.

[2] PANOFSKY, W. K.-PHILIPS, M. : Classical Electricity and Magnetism, Addison Wesley, Cambridge, 1955.

[3] JACKSON, J. D. : Classical Electrodynamics, Wiley, New York, 1962.

[4] LANDAU, L.-LIFŠIC, E. M. : Torija polja, GIFML, Moskva, 1962.

[5] ŠUMICHRAST, L. : Unified Approach to the Impulse Response and Green Function in the Circuit and Field Theory. Part II. Multi-Dimensional Case, JEEC 63 No. 6 (2012), 341-348.

[6] GRADŠTEJN, I. S.-RYŽIK, I. M. : Tablicy integralov, summ, rjadov i proizvedenij, GIFML, Moskva, 1963.

[7] ERDÉLYI, A. et al : Tables of Integral Transforms, vol. 2, McGraw Hill, New York, 1954.

[8] ŠUMICHRAST L': Field of moving point charge - application of generalized functions in the field theory (Pole pohybujúceho sa bodového náboja - aplikácia zovšeobecnených funkcií v teórii pol'a), Elektrotech. čas. 35 No. 10 (1984), 777-788. (in Slovak)

Received 17 February 2015

L'ubomír Šumichrast is with the Faculty of Electrical Engineering and Information Technology of the Slovak University of Technology since 1971, now holding the position of an Associate Professor and Deputy director of the Institute of Electrical Engineering. He spent the period 1990-1992 as a visiting professor at the University Kaiserslautern, Germany and spring semester 1999 as a visiting professor at the Technical University Ilmenau, Germany. His main research interests include the electromagnetic waves propagation in various media and structures, computer modelling of wave propagation effects as well as optical communication and integrated optics. 